\title{
Visual measurement of relative distances between three collinear dots rotating in a slanted plane
}

\author{
Johan Wagemans, Stefaan Tibau \\ Department of Psychology, University of Leuven, Tiensestraat 102, B-3000 Leuven, Belgium; \\ e-mail: johan.wagemans@psy.kuleuven.ac.be \\ Paper presented at the Applied Vision Association Conference/Workshop on Depth Perception, \\ Guildford, Surrey UK, 4 September 1997; revised version received 24 October 1998
}

\begin{abstract}
Displays consisted of orthographic and perspective projections of three collinear dots rotating rigidly around a fixed centre in a plane slanted $45^{\circ}$ in depth. Observers were asked to decide whether the middle of the three dots was exactly centred in 3-D space between the other two dots. The visible rotation segments were $120^{\circ}, 160^{\circ}$, or $200^{\circ}$ and the displacements were $2 \%$, $4 \%$, or $6 \%$. Our untrained observers performed more poorly overall than well-practised observers tested earlier by Lappin and Fuqua (1983 Science 221480 - 482). Results of additional manipulations suggested that 2-D image properties played a more important role than acknowledged by Lappin and Fuqua. First, displacement size and the position of the rotation centre produced nonlinear effects. Second, the direction of displacement affected performance. Third, projection type and display type (static snapshots versus elliptic traces) were involved in complex interactions. Clearly, performance was affected by 2-D image properties that should be irrelevant to the calculation of 3-D projective invariants.
\end{abstract}

\section{Introduction}

Lappin and Fuqua (1983) investigated how accurately the visual system can measure three-dimensional (3-D) distances undergoing perspective transformations. Observers were shown perspective and orthographic projections of three collinear dots rotating rigidly around a fixed centre in a plane slanted away from the observer. This stimulus configuration is shown in figure 1. Figure la shows a side view, in which the orthographic projection is indicated by the parallel grey lines. The perspective projection lines are black. A time-lapse image of the stimulus is shown in figure $1 \mathrm{~b}$ (with blackand-white reversal). Observers were asked to decide whether the middle of the three dots was exactly centred in 3-D space between the other two dots (ie a 3-D bisection task). The visible rotation segment in the slanted plane was $160^{\circ}$, rotating back and forth between $10^{\circ}$ and $170^{\circ}$ (ie start and end positions were located just above an imaginary horizontal line, at the right and left side of the screen, respectively). The slant could be $0^{\circ}, 30^{\circ}, 45^{\circ}$, or $60^{\circ}$. The displacements of the middle dot were $2 \%, 4 \%$, or $6 \%$ of the 3-D distance between the other two dots. Observers were always given feedback on a trial-by-trial basis and they were highly practised before they performed in the experiment.

Lappin and Fuqua (1983) obtained three important results. First, performance was very good, even comparable to two-dimensional (2-D) distance measurement. Second, no significant differences were found between different slants, not even when trials with different slants were randomly mixed together. Third, performance was equally good when stimuli were projected orthographically or perspectively. Lappin and Fuqua concluded from these results that observers were relying on 3-D structural invariants to perform the task. However, they did not specify what exactly it means 'to rely on 3-D structural invariants', and they left open what the underlying invariants that may have been used were.

These findings are remarkable. Under perspective projection, the 2-D distances between the three points as projected in the image $\left(a_{p}^{\prime}, b_{p}^{\prime}\right.$, and $c_{p}^{\prime}$ in figure 1a) change 

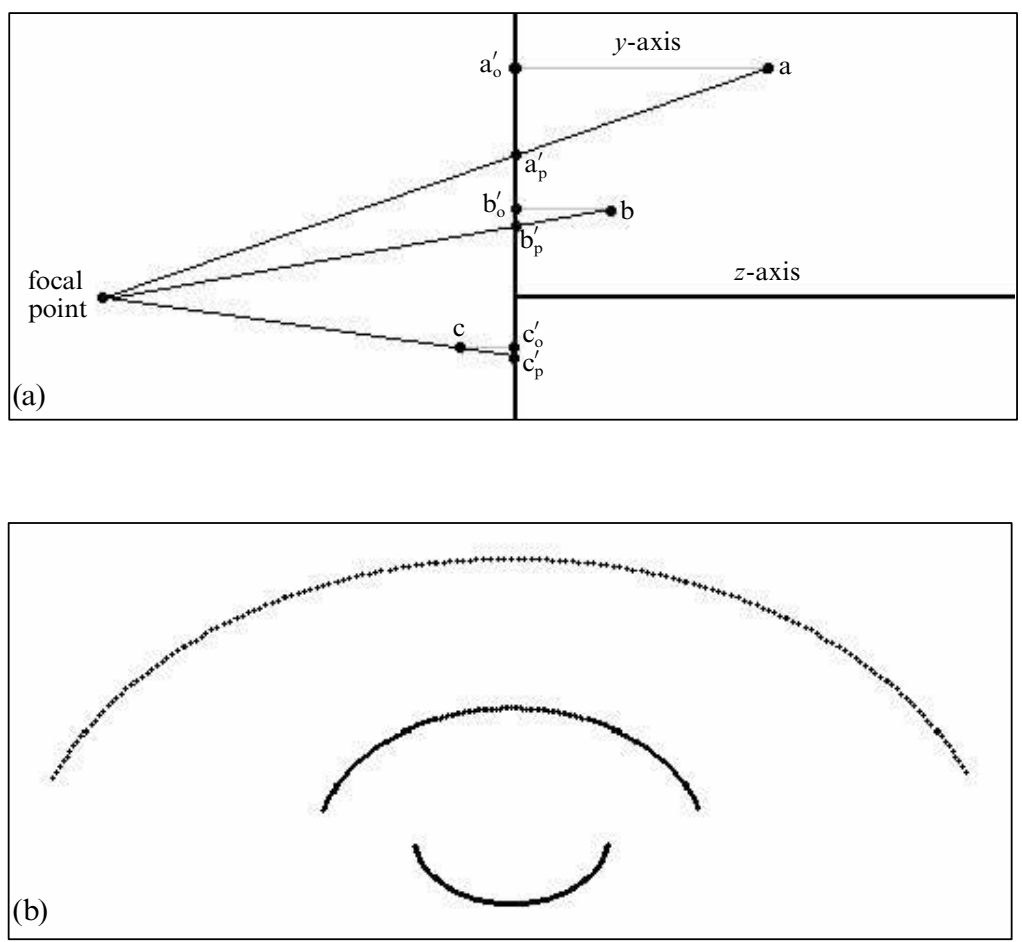

Figure 1. Basic stimulus configuration. (a) Side view. Orthographic projection is indicated by the parallel grey lines; perspective projection lines are black. Points in 3-D space, a, b, and c (from top to bottom, respectively), are projected onto points $\mathrm{a}^{\prime}, \mathrm{b}^{\prime}$, and $\mathrm{c}^{\prime}$ in the image, with subscript o standing for orthographically projected points and $\mathrm{p}$ for perspective ones. (b) A timelapse image of the stimulus viewed from the front as observers in our experiments did. The example is one with a $160^{\circ}$ segment without displacement (under perspective projection). Black and white have been reversed.

continuously when the dots are moving back and forth in the slanted plane. Thus, the deviation from bisection in the image with true 3-D bisection in space also changes with the orientation of the simulated line in the slanted plane. When the slant itself also changes, the problem is even more difficult. It is somewhat surprising that observers are able to estimate the 3-D distances with so little information about slant. Perhaps motion is crucial. Indeed, Johansson and Jansson (1968) have reported that observers sometimes perceive a 3-D rotating rod when only two points are shown with a continuously varying interpoint distance. Unlike in much other research, the difference between orthographic and perspective projection was substantial in Lappin and Fuqua's study (and ours), so that performance in the perspective condition could not be based on the preserved 2-D bisection under orthographic projection.

Moreover, these results seem to be in conflict with those obtained in more recent studies. For example, Todd and Bressan (1990) presented two line segments connected at the origin and rotating in depth about a vertical axis and they asked observers to indicate which of the two line segments appeared longer in 3-D space. ${ }^{(1)}$ Performance was only around $62 \%$ correct when one of the segments was $10 \%$ longer than the other one and a $75 \%$ correct level could only be reached with a $25 \%$ difference in length. Similarly low performance was obtained with a larger number of frames and with

(1) Note that Todd and Bressan (1990) used small-size stimuli and orthographic projection. For stimuli with a small depth range compared to the viewing distance, orthographic projection is a valid approximation of true perspective projection. In Lappin and Fuqua's (1983) study (and ours) it is not. 
other metric properties such as 3-D angles. In contrast, properties of so-called affine structure such as planarity and rigidity, were perceived much more accurately. The same difference between Euclidean properties and affine properties has since then been replicated many times with other stimuli, eg smoothly curved surfaces (eg Todd and Norman 1991); with other cues for 3-D structure, eg stereo (eg Tittle et al 1995); and even with real objects viewed directly in a natural environment (eg Norman et al 1996; but see Frisby et al 1996). This research has led to the conclusion that visually perceived structure from motion is invariant only up to arbitrary scalar transformations of distances in depth along the line of sight (eg Norman and Todd 1993; Norman et al 1996), a result which is congruent with theoretical analysis about so-called affine structure from motion (Koenderink and van Doorn 1991; but see also Pollick 1997).

Now, the fact that judgments of relative lengths of line segments oriented arbitrarily in 3-D space are inaccurate need not imply that Lappin and Fuqua's task should yield equally inaccurate performance. Indeed, bisection of a line segment or, more generally, judgments of relative lengths of parallel line segments, satisfies the so-called planarity constraint, shown to be theoretically sufficient for metric structure to be determined (Hoffman and Flinchbaugh 1982). In fact, Lappin and colleagues (Lappin and Ahlström 1994; Lappin and Love 1992) have subsequently shown that this planarity constraint is essential for making it possible to derive metric structure from motion. As a result, they have introduced the term intrinsic metric structure to refer to the scaling of spatial distances that preserves ratios of distances over changes in direction on a surface, which involves a maximum of three free parameters compared to six for metric distances in empty 3-D space.

In summary, visual measurements of relative 3-D lengths (and other metric properties) of line segments oriented arbitrarily in 3-D space seem to be inaccurate according to many recent studies, while visual measurements of relative 3-D lengths of coplanar line segments seem to be accurate according to Lappin and Fuqua's (1983) study. It thus appeared worthwhile to try to replicate Lappin and Fuqua's findings and test some additional conditions to try to delineate the range of the domain within which their conclusion is valid.

\section{Experiment 1: Replication with naive observers}

When we implemented the stimuli employed by Lappin and Fuqua (1983), our first impression was often one of nonrigid motion of a 2-D line segment shrinking and expanding while rotating in the frontoparallel plane. Only after a while could we see the moving dots as lying on a rigid line rotating in depth. This phenomenological observation made us wonder how much of Lappin and Fuqua's performance depended on the fact that they used highly trained observers in a paradigm with extensive feedback. In experiment 1 , we replicated their experiment with naive observers with feedback given only in the practice trials.

Another variable of interest in this experiment was the size of the rotation segment. Even in stimuli under perspective projection, the central dot would be exactly in the middle of the other two in the 2-D image itself, if the rotating line defined by the three dots were to pass the imaginary horizontal line (connecting $0^{\circ}$ and $180^{\circ}$ ). In other words, perhaps there was no need for accurate recovery of 3-D interpoint distances. Perhaps performance in Lappin and Fuqua's study was good because the locations of the dots at the start and end positions of a $160^{\circ}$ segment (ie at $10^{\circ}$ and $170^{\circ}$, respectively) provide a good enough approximation of the perfectly reliable locations with a $180^{\circ}$ segment. In a footnote, Lappin and Fuqua (page 480) mentioned that "performance was essentially unimpaired in a single rotation through an angle of $120^{\circ}$ " but the details of that experiment have not been published. 


\subsection{Methods}

2.1.1 Subjects. Subjects in experiment 1 were sixteen naive undergraduate students at the University of Leuven. All of them had normal or corrected-to-normal vision. They received partial course credit for their participation.

2.1.2 Apparatus. Stimuli were generated by a computer program written in $\mathrm{C}$ that ran on a PC with a $80486 \mathrm{DX}$ processor and a clock speed of $120 \mathrm{MHz}$. They were displayed on a VGA computer screen with $640 \times 480$ pixel spatial resolution and $60 \mathrm{~Hz}$ temporal resolution. Responses were given by pressing the left arrow or the right arrow on the keyboard. Assignment of response keys to positive ("yes, it is in the middle") and negative ("no, it is displaced") responses was counterbalanced across subjects.

2.1.3 Stimuli. As in Lappin and Fuqua's (1983) study, three collinear dots (filled circles with a radius of 1 pixel each) were rotating back and forth in a slanted plane: As soon as the dots appeared at the right side of the screen, they started to rotate to the left and back to the right, where the screen turned black as soon as the dots arrived at their original positions. The angular velocity of the dots was approximately $100^{\circ} \mathrm{s}^{-1}$. The dots were white $\left(29.5 \mathrm{~cd} \mathrm{~m}^{-2}\right)$ on a black background $\left(2.8 \mathrm{~cd} \mathrm{~m}^{-2}\right)$.

We did not manipulate the slant, but kept it fixed at $45^{\circ}$. Instead, we varied the size of the rotation segment to have an internal angle of $120^{\circ}, 160^{\circ}$, or $200^{\circ}$. This was done by starting with a line segment oriented at, respectively, $30^{\circ}, 10^{\circ}$, and $-10^{\circ}$ (with $0^{\circ}$ at the right position of the horizontal axis and positive angles being counterclockwise rotations) and ending at, respectively, $150^{\circ}, 170^{\circ}$, and $190^{\circ}$ (with $180^{\circ}$ at the left position of the horizontal axis). We will call this factor segment size. Other details remained as in Lappin and Fuqua's study: displacement of the middle dot could be $2 \%, 4 \%$, or $6 \%$ of the distance between the two other dots (called displacement size) and projection could be orthographic or perspective (called projection type).

The distance between the image plane and the focal point in stimuli under perspective projection was $2^{1 / 2}$ times the length between the two extreme dots. This gives considerable perspective distortion. The size of the stimuli depends on segment size and projection type. Our largest stimuli were those under orthographic projection with a segment size of $200^{\circ}(5 \mathrm{deg} \times 12 \mathrm{deg}$ of visual angle). Our smallest stimuli were those under perspective projection with a segment size of $120^{\circ}(4 \mathrm{deg} \times 9 \mathrm{deg})$. The experiment was conducted in a dimly lit room and the subjects looked monocularly (with the nondominant eye patched) at the stimuli from an average distance of $80 \mathrm{~cm}$.

2.1.4 Procedure. When the displays were first shown, observers were told that these moving dots could be seen as lying on a rigid line sweeping across a slanted plane, very much like a wiper on a slanted windscreen. As soon as they confirmed seeing the stimulus in this way, observers were instructed to judge for each subsequent trial whether or not the middle dot was exactly centred between the other two dots in 3-D space. Half of the observers saw orthographic projections of the stimuli, while the other half received the perspectively projected stimuli. Each observer received nine blocks of 52 trials, each block consisting of one of three segment sizes and one of three displacement sizes. All subjects started with the larger displacements, followed by the intermediate and smaller ones, while the order of segment sizes was random. In each experimental condition, there were 52 stimuli to be judged, half of which showed a displacement of the middle dot in 3-D space. When the middle dot was displaced, this could be in two directions. In half of the trials with displacement, the middle dot was displaced in the direction towards the viewer (ie downwards on the computer screen); and in the other half, it was displaced in the direction away from the viewer (ie upwards on the computer screen). In section 2.2, we will also refer to these as negative and positive displacements, respectively. 
Before starting with the experimental trials, subjects saw six randomly chosen stimuli per condition as practice trials. During this practice phase, subjects received immediate feedback by means of an auditory signal (a $250 \mathrm{~ms}$ beep at $440 \mathrm{~Hz}$ to indicate an incorrect response). In the test phase, this immediate feedback was no longer provided. After each block of 52 trials, only the overall percentage of correct trials was displayed. Such global feedback does not allow subjects to improve performance on a trial-by-trial basis but helps them to remain motivated. The duration of each experimental session was approximately $1 \mathrm{~h}$.

\subsection{Results}

Because casual inspection of the means showed that there were considerable differences between the forward (positive) and backward (negative) displacements, we did not analyse our data as Lappin and Fuqua (1983) did, by collapsing forward and backward displacements and computing response-bias-free measures based on separate hits and false-alarm rates for each of the three displacement sizes. Instead, we kept the two displacement directions separate and computed average percentages of correct responses for each of seven conditions of the recoded variable, displacement, $-6 \%,-4 \%,-2 \%, 0 \%,+2 \%$, $+4 \%$, and $+6 \%$. In all cases, we have also computed $d^{\prime}$ by collapsing forward and backward displacements but we will only report these when the results are different from those with the percentage of correct responses.

We analysed our data using analysis of variance (ANOVA) in a mixed design with one between-subjects variable (projection type) and two within-subjects variables (displacement and segment size). Projection type did not yield a significant effect $\left(F_{1,14}=1.47\right.$, $p>0.2)$, nor did segment size $\left(F_{2,28}<1\right)$. Displacement, however, did show an effect $\left(F_{6,84}=3.37, p<0.01\right)$. We also observed a marginally significant interaction effect of displacement with projection type $\left(F_{6,84}=2.03, p=0.07\right)$.

As can be seen in figure 2, observers' performance was almost equal for both directions of displacement when judging perspectively projected stimuli. The function for perspective stimuli, indicated by filled squares connected by a thick line, was U-shaped. This was not the case for orthographically projected stimuli (open diamonds connected by a thin line). Here, performance was poorer for displacements away from the viewer (ie with positive sign) than for displacements towards the viewer (ie with negative sign).

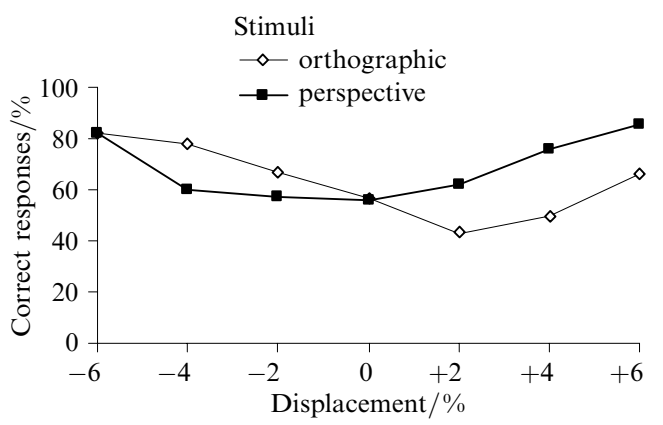

Figure 2. Results from experiment 1. Interaction effect of displacement and projection type on percentage of correct responses.

Finally, there was also a three-way interaction of displacement $\times$ projection type $\times$ segment size $\left(F_{12,168}=2.77, p<0.005\right)$. As can be seen in figure 3 , the basis of this interaction is the fact that segment size had almost no effect on the orthographic stimuli (figure 3a), while the average U-shaped function of displacement on the percentage of correct responses for the perspective stimuli was modulated quite strongly by the effect of segment size (figure $3 b$ ). We will return to this effect in the general discussion (section 5). 


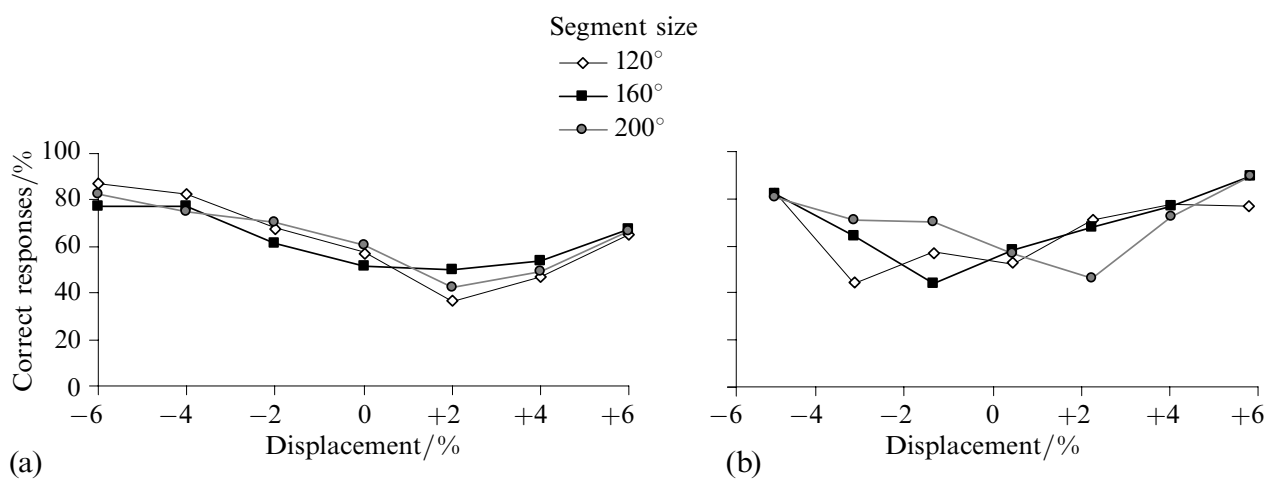

Figure 3. Results from experiment 1 . Displacement $\times$ projection type $\times$ segment size interaction effect on the percentage of correct responses. (a) For orthographic stimuli, segment size has little or no effect. (b) For perspective stimuli, segment size interacts strongly with displacement.

\subsection{Discussion}

In general, our naive observers performed more poorly overall than Lappin and Fuqua's (1983) trained subjects. More importantly, there were no overall effects of segment size and of projection type. Both results corroborate Lappin and Fuqua's conclusion that observers do not simply rely on 2-D distances in the image. If this were the case, performance should have declined with decreasing segment size and performance should have been much better with orthographic projection than with perspective projection.

However, two aspects of our data raise some doubts about the use of 3-D structural invariants as the source of information underlying task performance. First, as can be seen clearly in figure 3, performance was not a perfectly linear function of displacement size (ie expressed in relation to 3-D distance between the two extreme dot positions). One reasonable interpretation of performance being based on invariants would be to expect a linear dependence of performance on the 3-D displacement which was manipulated in equal steps of $2 \%$. Second, for orthographically projected stimuli there was a difference between displacements toward and away from the observer.

This can be interpreted as follows (see figure la again). Let us indicate the three dots from top to bottom in the slanted plane by a, b, and c, and their projected counterparts in the image by $\mathrm{a}^{\prime}, \mathrm{b}^{\prime}$, and $\mathrm{c}^{\prime}$, respectively. When the central dot is located exactly in the middle of the two other dots in 3-D space, the interdot distances in the image, $\left|\mathrm{a}^{\prime} \mathrm{b}^{\prime}\right|$ and $\left|\mathrm{b}^{\prime} \mathrm{c}^{\prime}\right|$, are equal under orthographic projection, while $\left|\mathrm{a}^{\prime} \mathrm{b}^{\prime}\right|$ is shorter than $\left|b^{\prime} c^{\prime}\right|$ under perspective projection. This is because in 3-D space dot $\mathrm{c}$ is located closer to the observer than dot $\mathrm{b}$ and this in turn is closer than dot a (ie because the top side of the plane is always slanted away from the observer). In the case of positive displacements, away from the observer, under orthographic projection $\left|\mathrm{b}^{\prime} \mathrm{c}^{\prime}\right|$ will become longer than $\left|a^{\prime} b^{\prime}\right|$, which the observer might interpret as resulting from dot $b$ being exactly centred between dot a and dot $\mathrm{c}$ under perspective projection. This will lead to a propensity to (incorrectly) respond "yes, it is in the middle". In the case of negative displacements, towards the observer, $\left|\mathrm{b}^{\prime} \mathrm{c}^{\prime}\right|$ will always be shorter than $\left|\mathrm{a}^{\prime} \mathrm{b}^{\prime}\right|$, which can never correspond to dot $\mathrm{b}$ exactly centred between dot a and dot $\mathrm{c}$ in 3-D space. This will lead to a propensity to (correctly) respond "no, it is displaced". Because for perspective stimuli $\left|\mathrm{b}^{\prime} \mathrm{c}^{\prime}\right|$ was also a bit shorter than $\left|\mathrm{a}^{\prime} \mathrm{b}^{\prime}\right|$ in some conditions, a similar strategy may have been used there. We will return to this issue in more detail in the general discussion (section 5).

In sum, the data from experiment 1 suggest that performance was probably not based completely on either the 2-D distances in the image or invariant 3-D structure as such. Instead a mixture of strategies may have been used. 


\section{Experiment 2: Effects of the centre of rotation}

Now that we have replicated some of the most important results from Lappin and Fuqua's (1983) study, we could start to investigate in more detail what sort of information may underlie task performance. What might Lappin and Fuqua (1983, page 482) mean when they say that "the geometric information for this discrimination must have been some aspect of the 3-D structure that remained invariant under projective transformations"? If the invariant properties must be invariant under the most general group of transformations which is relevant to the experimental circumstances, it must be projective invariants. The best known projective invariant is the cross-ratio, which has already been shown to be useful in other perceptual tasks (Cutting 1986). However, the cross-ratio is defined on four collinear points and only three are present in the experimental stimuli in Lappin and Fuqua's as well as our study. It has been shown before that some tasks which seem to require four points as minimal information can also be done with three points (Simpson 1986; Wagemans et al 1998) but it remains true that four points would be needed if performance were based exclusively on using cross-ratios.

If observers were able to extract the rotation centre, which is always a fixed point collinear with the three stimulus dots, it could have been used as the fourth point needed to compute cross-ratios. To assess whether the rotation centre somehow played a role in the way observers performed the task, we manipulated its location in experiment 2. In one condition, the lowest point on the screen was used as the centre of rotation (and thus it was stationary). This leads to a singularity in the cross-ratios and may be anticipated to strongly influence performance if cross-ratios were computed with the rotation centre as the fourth point.

\subsection{Method}

3.1.1 Subjects. Sixteen new subjects from the same pool of naive subjects participated in this experiment.

3.1.2 Stimuli. Stimuli in this experiment were essentially the same as in experiment 1. Because segment size did not play a role in experiment 1 , it was fixed at $160^{\circ}$, as in Lappin and Fuqua's (1983) original experiment. Instead, we varied a new factor, the position of the centre of rotation relative to the three dots (called centre of rotation). In practice, we did not move the centre of rotation relative to the three dots but we moved the three dots relative to the centre of rotation (see figure 4). In this way, the centre of rotation stayed exactly in the middle of the computer screen for all conditions. ${ }^{(2)} \mathrm{We}$ indicate the five

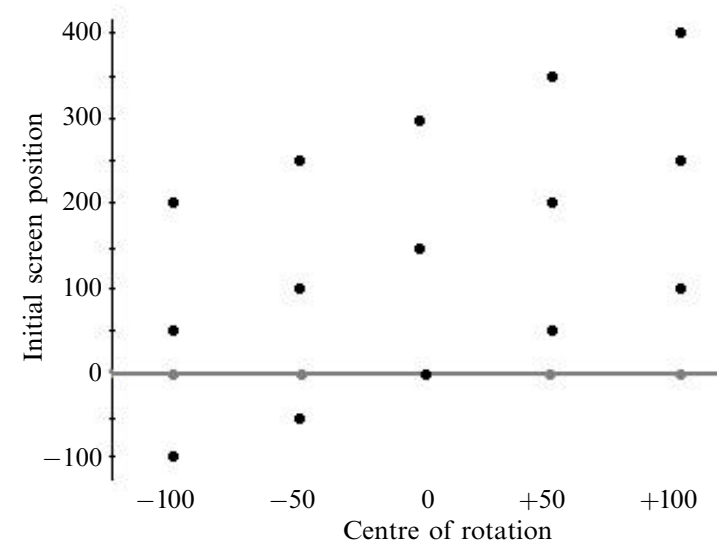

Figure 4. Indication of how the position of the centre of rotation was manipulated in experiment 2. The five possible locations of the centre of rotation are indicated by grey dots; the fact that these are all situated on a horizontal line indicated by 0 at the left illustrates that the displays were always centred around the centre of rotation; the stimulus points (indicated by black dots) were shifted higher on the screen when the position of the centre of rotation changed from -100 at the left of this figure to +100 at the right (eg from 200 to 400 for dot a). In experiment 2 , all five positions were tested; in experiment 3 only $-100,0$, and +100 .

(2) This procedure implies that the eccentricity of the dots varies with the location of the centre of rotation. However, the results will indicate that this has had no detrimental effect on performance. The conditions with the most eccentric dots were not systematically more difficult (on the contrary, they were amongst the easiest). 
centre of rotation conditions by the screen coordinates of the lowest point: $-100,-50,0$, $+50,+100$. Important here is that in conditions -100 and -50 the dots are not moving in a uniform direction. When the lower dot is moving from the left to the right, the other two dots are moving from the right to the left and vice versa.

Given the fact that in this experiment other centres of rotation were used (compared to experiment 1) and segment size was fixed at $160^{\circ}$, the range of sizes of the stimuli also changed. In this experiment the largest stimuli were those under orthographic projection, with centre of rotation at $+100(7 \mathrm{deg} \times 19 \mathrm{deg})$. The smallest stimuli were those under perspective projection with the centre of rotation at $-100(4.5 \mathrm{deg} \times 9 \mathrm{deg})$.

3.1.3 Procedure. Because we had more conditions in this experiment and subjects in experiment 1 already complained about tired eyes and boredom, we lowered the number of trials per condition to 20 stimuli, 10 where the central dot was exactly in the middle of the other two and 10 where it was displaced ( 5 toward the observer and 5 away from the observer). The order in which the 15 blocks of trials ( 3 displacement sizes $\times 5$ centres of rotation) were presented was random for all observers. As in experiment 1, eight participants received orthographically projected stimuli and the other eight received perspectively projected ones. The rest of the procedure also remained the same.

\subsection{Results}

As expected on the basis of Lappin and Fuqua (1983) and our experiment 1, projection type yielded no effect on the percentage of correct responses $\left(F_{1,14}<1\right)$, while displacement did yield a reliable effect $\left(F_{6,84}=23.44, p<0.01\right)$. The interaction between projection type and displacement, which was marginally significant in experiment 1 , now turned out to be statistically reliable $\left(F_{6,84}=11.74, p<0.01\right)$. As can be seen in figure 5 , the trends causing this interaction were the same: For perspective stimuli, there was little or no difference between the two displacement directions, while displacements away from the observer were more difficult than displacements towards the observer for orthographic stimuli. This was supported statistically by reliable a posteriori comparisons (using Tukey's HSD) for 2\% $(p<0.0005), 4 \%(p<0.0005)$, and $6 \%(p<0.05)$.

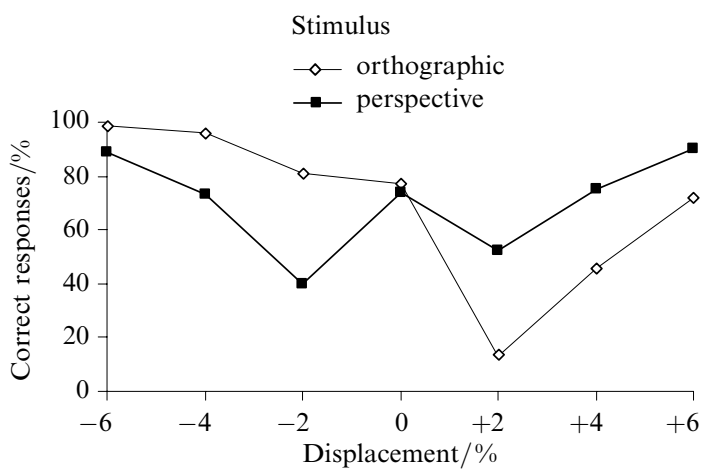

Figure 5. Results from experiment 2. Interaction effect of displacement and projection type on percentage of correct responses.

Centre of rotation produced significant differences on $d^{\prime}$ (although its effect on the percentage of correct responses failed to reach statistical significance) $\left(F_{4,56}=2.77\right.$, $p<0.05)$, an effect which was further modified by an interaction with projection type $\left(F_{4,56}=3.01, p<0.05\right)$. As shown in figure 6 (and confirmed statistically by a posteriori comparisons), the largest difference was between -50 and +50 positions for the orthographic stimuli. 


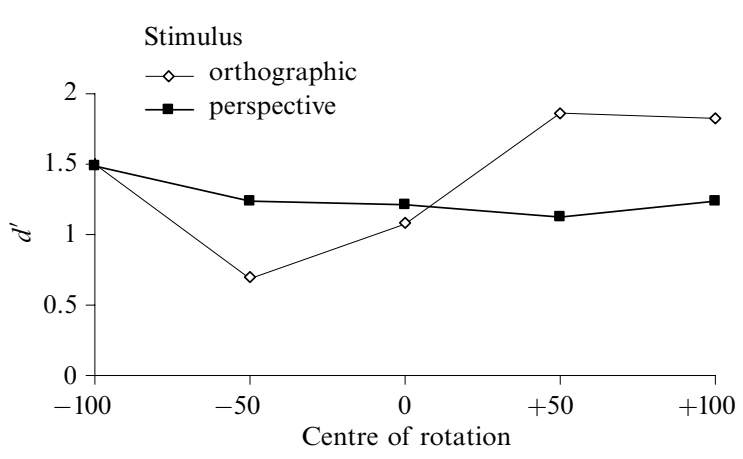

Figure 6. Results from experiment 2. Interaction effect of centre of rotation and projection type on $d^{\prime}$.

\subsection{Discussion}

First of all, the most surprising finding from experiment 1, the effect of displacement direction for orthographic stimuli, was replicated in experiment 2, although observers were generally more accurate in experiment 2 than in experiment 1 . The effect of centre of rotation tells us that performance may have been poorer in experiment 1 because the most difficult position of the centre of rotation has been used there.

Indeed, as can be inferred from figure 6 , the position called -50 was clearly most difficult for orthographic stimuli. This can be explained as follows. First, when the centre of rotation lies inbetween the three stimulus dots (ie with negative positions of the centre of rotation), one dot moves in a direction opposite to the direction of the other two dots. As could be anticipated, this more complicated motion pattern may have been more difficult to organise into a strong unified percept for at least some observers on some of the trials. Second, this opposite motion direction may have been especially confusing when the lower stimulus dot was relatively close to the rotation centre (at -50$)$. When the lower stimulus dot was further away (at -100$)$, it was probably segregated from the other dots anyway. Under grouping by proximity (Kubovy et al 1998; Kubovy and Wagemans 1995), the lowest dot in the -50 condition could be grouped with the centre of rotation and through it with the other two dots located higher in the slanted plane, but the opposite motion direction may have counteracted that tendency. This competition in terms of grouping principles (proximity versus common motion direction) may have yielded very unstable percepts.

Perception of perspectively projected stimuli was not affected by the position of the centre of rotation. This is probably because in that case we have a far more realistic appearance of the stimulus as a line segment moving in depth. Here, differences in depth are translated to differences in distance in the image plane. Because we do not have such a mapping in orthographic projections, 2-D image characteristics instead of 3-D appearance may have been more important for perceptual groupings in the orthographic displays.

\section{Experiment 3: The effect of different display types}

The fact that the location of the centre of rotation did not have an influence on performance with perspectively projected stimuli was against its alleged role as a fourth point allowing computation of the cross-ratios which are known to be projectively invariant. While one would have expected radically different performance in the condition with the lowest point as the centre of rotation (with singular cross-ratios), it did not differ reliably from the other conditions. This raises the question about other possible sources of information that could have been used.

As argued previously (Van Gool et al 1994), many invariants have been identified by mathematicians and implemented successfully in working computer vision systems that are not widely known amongst perception researchers. It is useful to distinguish between 
at least two types of invariants: algebraic invariants based on point coordinates and differential invariants based on curvature (see also Carlsson et al 1996; Forsyth et al 1991). Algebraic invariants other than cross-ratios can be defined for Lappin and Fuqua's (1983) as well as our stimulus displays. If one assumes that the dot positions could be stored for separate salient frames from the motion sequence (eg the initial and final frames), non-collinear five-point equivalents for the collinear four-point cross-ratios could be computed that are also algebraic projective invariants (see Van Gool et al 1994, for more details). For differential projective invariants, one has to make the opposite assumption, namely that different frames from the motion sequence can be integrated to have continuous measures of the trace of each dot. These traces, which are elliptic in the case of perspective stimuli, form conic sections which are used frequently in many recent computer vision techniques for curved object recognition from different viewpoints (eg Carlsson et al 1996; Forsyth et al 1991). Niall (1998) has recently obtained rather accurate estimates of 3-D invariants from pairs of rotating ellipses.

In experiment 3, we wanted to increase the likelihood that these projective invariants are used, by improving the circumstances for their extraction. We reasoned that the extraction of algebraic invariants would become easier by showing only static snapshots of the original continuously moving stimulus. Alternatively, extracting differential invariants would become easier by showing the motion paths of the dots explicitly as a trace left on the screen.

\subsection{Methods}

4.1.1 Subjects. Because the design of this experiment implied a larger number of trials per observer, we decided to use a smaller number of observers. Four subjects participated in this experiment. Two of them were naive about the purposes of the experiment; the other two were the authors of this paper.

4.1.2 Stimuli. Once again we employed variations of the same stimuli. Five stimulus characteristics were varied. Four of these had been used in one or both of the previous experiments. The factors projection type and displacement remained as before. In addition, we included a smaller sample from the major variable in experiment 1, segment size $\left(120^{\circ}\right.$ or $\left.160^{\circ}\right)$, and from the major variable in experiment 2 , centre of rotation $(-100,0$, or +100 ; see figure 4 again).

The most important, new manipulation was what we call display type. In the first condition (original), the original stimuli were used again: three continuously moving dots. In the second condition (snapshot), subjects were shown only five static snapshots of $750 \mathrm{~ms}$ each, separated by a black screen during $25 \mathrm{~ms}$, in the following sequence: one at the right, one in the middle, one at the left, one in the middle again, and one at the right again. In the third condition (ellipse), the display showed three dots, continuously moving, while they left their traces behind (similar to figure 1b). The dots moved first from the right to the left. Then the screen turned black and immediately afterwards the opposite movement was shown. The size of the stimuli varied between $4.5 \mathrm{deg} \times 7.5 \mathrm{deg}$ (perspective projection, centre of rotation at -100 , segment size of $120^{\circ}$ ) and $7 \mathrm{deg} \times 19 \mathrm{deg}$ (orthographic projection, centre of rotation at +100 , segment size of $160^{\circ}$ ).

4.1.3 Procedure. To reduce the difference in level of expertise between the observers, the following two measures were taken. First, we explained to the naive observers the difference between orthographic and perspective projection. For example, they were told that relative distances are preserved under orthographic projection but not under perspective projection because of perspective distortion. In this way, we could verify empirically the explanation we gave to the interaction effect between projection type and displacement in experiments 1 and 2 . In the present experiment, this interaction effect should disappear, because now subjects will no longer misinterpret forward-displaced 
orthographic stimuli as centred perspective stimuli. Second, because of the fact that the naive subjects did not have any experience with the kind of stimulus we used, we let them practice with the original stimuli during $1 \mathrm{~h}$. The authors of this paper did not go through this practice session because they already had enough experience in working with the stimuli. After that, six sessions (one for every combination of projection type and display type) of $1 \mathrm{~h}$ each followed.

As in the previous experiments, every session contained a practice phase (which all of the subjects had to go through) and a test phase. During the practice phase (with feedback), the subjects were shown five randomly selected trials per condition of the session they had to go through. Then the test phase (without feedback) followed. In every session, this phase contained 18 conditions ( 3 displacement sizes $\times 2$ segment sizes $\times 3$ centres of rotation), in a completely randomised order, each of which contained 20 trials (10 without displacement, 5 with forward displacement, and 5 with backward displacement).

\subsection{Results}

As in the previous experiments, displacement size had a large effect on performance, both on the percentage of correct responses $\left(F_{2,6}=105.83, p<0.0001\right)$ and on $d^{\prime}$ $\left(F_{2,6}=28.04, p<0.001\right)$. A posteriori analyses showed that $2 \%$ displacements yielded lower performance $(71 \%$ or 1.40$)$ than either $4 \%(87 \%$ or 2.36 , respectively) or $6 \%$ (92\% or 2.63 , respectively) displacements, which differed reliably from one another only for the percentage of correct responses. When centre of rotation was +100 , performance (the percentage of correct responses) was somewhat better $\left(F_{2,6}=5.25, p<0.05\right)$. More importantly, display type also had a reliable effect on performance, both on the percentage of correct responses $\left(F_{2,6}=6.54, p<0.05\right)$ and $d^{\prime}\left(F_{2,6}=8.45, p<0.05\right)$. A posteriori analyses showed that snapshot stimuli were somewhat easier $(88 \%$ or 2.85$)$ than either original stimuli ( $83 \%$ or 1.77 , respectively) or ellipse stimuli $(79 \%$ or 1.77 , respectively).

Many of the interactions between these significant main effects also turned out to be reliable. The most interesting of these are the display type $\times$ centre of rotation interaction $\left(F_{4,12}=5.57, p<0.01\right)$, caused by +100 centres yielding a significant advantage only for the ellipse stimuli (see figure 7a), and the display type $\times$ displacement interaction $\left(F_{12,36}=2.53, p<0.05\right)$, caused by ellipse stimuli yielding lower performance than the other two display types, only for backward (negative) displacements, not for forward (positive) displacements (see figure $7 \mathrm{~b}$ ). As anticipated, the projection type $\times$ displacement interaction did not reach statistical significance $\left(F_{6,18}=1.27, p>0.3\right)$.

$$
\begin{aligned}
& \text { Condition } \\
& \multimap \text { original } \\
& \rightarrow \text { ellipse } \\
& \multimap \text { snapshot }
\end{aligned}
$$

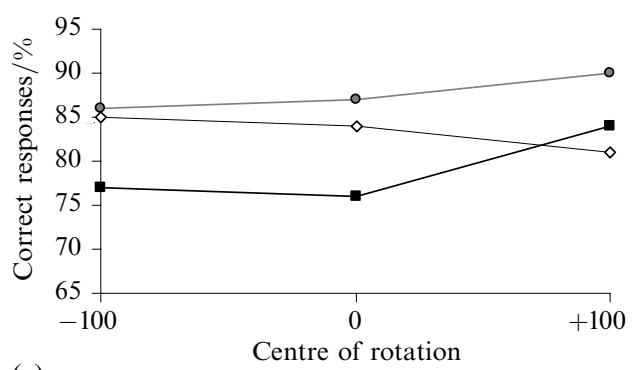

(a)

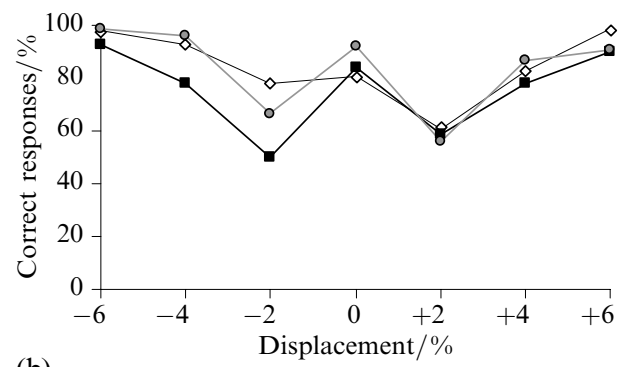

(b)

Figure 7. Results from experiment 3. (a) Interaction effect of display type and centre of rotation on the percentage of correct responses. (b) Interaction effect of displacement and display type on the percentage of correct responses. 


\subsection{Discussion}

Experiment 3 was designed in an attempt to determine which of two types of invariants have most likely been used by observers when performing the task of judging whether a midpoint bisects a line segment into two halves of equal 3-D length. Although there was a small and statistically significant advantage for the snapshot stimuli, which were supposed to facilitate the extraction and use of algebraic invariants based on point coordinates, for many conditions performance with the ellipse stimuli and even the original stimuli was just as good. In fact, the most important finding from this experiment was that display type interacted with most of the other experimental variables, suggesting a large degree of flexibility in the extraction and use of multiple sources of information, depending on the particular display conditions.

\section{General discussion}

In our effort to understand the results from Lappin and Fuqua's (1983) study, showing excellent performance in judgments of relative 3-D distances between three collinear dots rotating in a slanted plane, we have been able to replicate some of their most important findings, while at the same time obtaining additional findings that seem to qualify their conclusion about the use of 3-D structural invariants under perspective transformations.

We have replicated that observers can do the task just as easily with perspectively projected stimuli as with orthographically projected ones. We have also replicated that task performance is unaffected by the size of the segment of the circular motion path of the dots sweeping about a fixed centre. Both of these variables, projection type and segment size, should have yielded significant differences if observers were to map the 2-D distances on the screen literally onto 3-D distances in space.

However, in each of the three experiments, we observed at least one effect which is not compatible with the idea that subjects' performance is based completely on their using 3-D structural invariants as such. If subjects were using invariants, then, first, the direction of displacement should not matter to the calculation of these invariants and, second, the position of the centre of rotation should not matter either. In all experiments, when they were included as experimental variables, these factors were involved in reliable interaction effects. Moreover, all of our attempts to directly influence the extraction and use of a particular invariant (cross-ratio in experiment 2) or of a particular class of invariants (algebraic versus differential in experiment 3 ) have failed to confirm the superiority of one experimental condition over all others, which would reveal the 3-D structural invariants underlying subjects' performance.

Instead, we think that in different conditions different sources of information become more salient or more reliable. Observers have most likely been using an opportunistic mixture of strategies instead of just one, picking out the source of information that happened to be most salient, most reliable, and thus most useful, in each particular display condition. Previous research on similar problems has already indicated that it is often very difficult to pinpoint one single source of information as the basis for human performance. For example, in the context of Todd's affine structure (eg Norman and Todd 1993; Norman et al 1996; Tittle et al 1995; Todd and Bressan 1990; Todd and Norman 1991), others have demonstrated that 2-D Euclidean properties are also important (eg Eagle and Blake 1995; Hogervorst and Eagle 1998; Pollick 1997). In the context of Lappin's intrinsic metric structure (eg Lappin and Ahlström 1994; Lappin and Love 1992), others have argued that 2-D image cues are at least equally important (eg Pizlo and Salach-Golyska 1994). Similar debates have been going on with respect to affine shape equivalence (Kukkonen et al 1996; Wagemans et al 1998) and about retinal versus optic flow (eg Cutting 1996; Cutting et al 1992; Kim et al 1996). 
More generally, while some have argued that psychophysical research so far has failed to evaluate unambiguously the specific geometric relations that are used to perceive 3-D structure from motion (Sperling et al 1989, 1990), others have stressed that 3-D geometric relations always have their counterparts in the 2-D image and that it is somewhat arbitrary to call these 2-D image properties information or artifacts (Braunstein and Todd 1990). To us, it seems best to consider all possible sources of information and try to relate performance to them to assess their relative usefulness in different contexts (a position which is somehow tributary to Cutting's position about so-called 'directed perception'; see Cutting 1986, 1991).

In the context of our research, the cue ${ }^{(3)}$ that seems most relevant is the length ratio of the two collinear line segments between the dots. This is an affine invariant, which means that in our stimulus under orthographic projection it has a constant value, even when the slant of the stimulus changes. This is not the case under perspective projection. Even when the slant is kept constant, as in our research, the length ratio $\left(\left|a^{\prime} b^{\prime}\right| /\left|b^{\prime} c^{\prime}\right|\right.$, see figure la again) changes continuously when the line is moving from near-horizontal positions in the slanted screen (with little perspective distortion) to vertical positions (with maximal distortion). We have plotted the function of length ratio against orientation in the plane for the different displacement conditions (see figure 8 ). When the middle dot is centred exactly between the two extreme dots (in 3-D space), the length ratio is 1 only at $0^{\circ}$ and $180^{\circ}$. As indicated earlier, this means that at these positions the lengths of the two segments (or the two pairwise distances) are equal in the image screen and it would be easy to respond "yes". As soon as the line segment moves away from these screen orientations, perspective distortion can become considerable (eg $\left|\mathrm{a}^{\prime} \mathrm{b}^{\prime}\right| /\left|\mathrm{b}^{\prime} \mathrm{c}^{\prime}\right|<0.79$ at $90^{\circ}$ ) and it is a much more difficult task for observers to determine whether the length ratio is just about small enough to correspond to no 3-D displacement. Negative displacements (ie towards the viewer) are sometimes easy to notice because the upper segment $\left|a^{\prime} b^{\prime}\right|$ becomes visibly longer than the lower segment $\left|b^{\prime} c^{\prime}\right|$ (ie $\left|a^{\prime} b^{\prime}\right| /\left|b^{\prime} c^{\prime}\right|>1$ ) for a large sample of the line segment orientations along its motion path, which can never correspond to a central position in 3-D space. Positive displacements (ie away from the viewer) will lead to good performance when the upper segment $\left|a^{\prime} b^{\prime}\right|$ becomes much shorter than the lower segment $\left|b^{\prime} c^{\prime}\right|$ for it to possibly correspond to the proper ratio with no displacement.

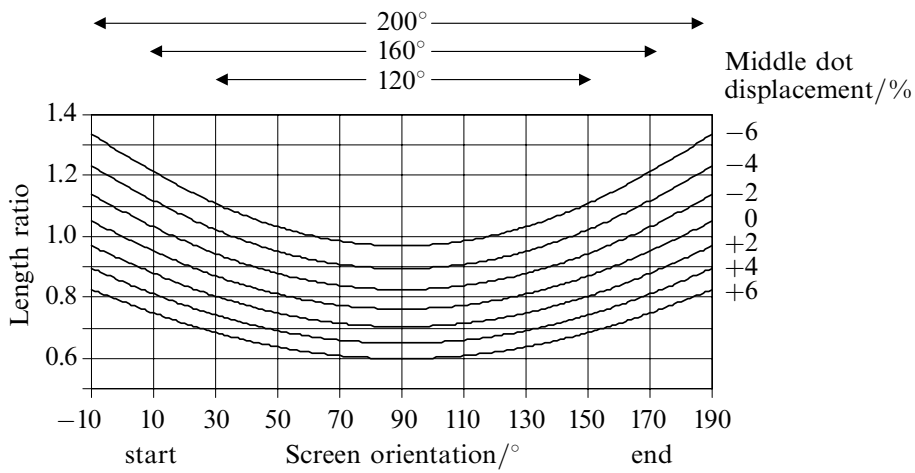

Figure 8. Length ratio $\left|\mathrm{a}^{\prime} \mathrm{b}^{\prime}\right| /\left|\mathrm{b}^{\prime} \mathrm{c}^{\prime}\right|$ as a function of different orientations of the virtual line segments in the plane, for all of the displacement conditions. Several properties of these functions correspond to several noticeable changes in performance levels (see text for more details).

(3) We use the term cue as a general concept, supposed to embrace perfectly reliable, true 3-D invariants, as well as imperfectly reliable, 2-D image properties correlated with what happens in 3-D space. In this way, we remain theoretically neutral in the sense that we have no a priori ideas about what source of information should be used by observers. 
It seems possible to relate performance in the perspective projection conditions to the size of this length ratio. Let us take the data from experiment 1 as an example (see figure $3 b$ ). The interaction between displacement and segment size seemed rather complicated for perspective stimuli. For each of the three segment size conditions, the displacement condition producing the lowest performance level was different. For 200 segments, performance was generally high for negative displacements, probably because the length ratio was close to 1 or higher for a significant sample of the motion path, leading to relatively easy correct rejections. With positive displacements, performance was minimal for $+2 \%$ displacements. Many more incorrect 'yes' responses are made here perhaps because the length ratio at the start and end positions is close to that of the condition without displacement. For $160^{\circ}$ segments, performance was generally high except for $-2 \%$ displacements where length ratios at the extreme positions are again confusable with those without displacement. For $120^{\circ}$ segments, both $-4 \%$ and $-2 \%$ displacements led to relatively large false-alarm rates.

In sum, displacements were relatively easy to detect when the length ratio was close to 1 over a considerable range of line segment positions along the motion path. When this cue was not available, subjects may have relied more on the length ratio at the start and end positions which may have led to more false alarms when the ratio was confusable with that of the condition without displacement.

In addition to the length ratio, there is a whole range of other cues. For example, several curvature parameters are present in the stimulus, some of which are absolute invariants and some are only relative invariants under perspective transformations (see Foster and Simmons 1994; Foster et al 1993; Foster and Wagemans 1993, for more details). In this research by Foster and colleagues, geometric attributes of curved contours (such as the sag, the area enclosed by the curve, and the chord joining its ends) were examined for invariance of the Weber fraction for a curvature discrimination task under symmetry-preserving affine transformations. It was shown that only some of these attributes satisfied the invariance condition.

A property of a fundamentally different nature is the velocity of the middle dot. This depends on the length of the trajectory travelled by each dot, quantified by the arc length of the curved trace. For each position of the middle dot, velocity increases when the position shifts from locations at the lower edge of the slanted plane $(-6 \%$, $-4 \%, \ldots)$ to the higher edge of the slanted plane $(\ldots,+4 \%,+6 \%)$, because the dot has to complete a longer trajectory in the same amount of time. Related to this is the density (or luminance) of the central curve. In each display, every curve consists of an equal number of dots, shown one at a time. For example, a curve with a rotation segment of $160^{\circ}$ consists of 161 dots, shown one at a time, one at every degree on the curve from $10^{\circ}$ to $170^{\circ}$. So, the farther away a curve is relative to the observer, the longer is the arc length that has to be drawn with a constant number of points. In consequence, the density (and with it also the luminance) of the curves gets lower with increasing distance from the observer. This is a rather salient property in some of the display conditions.

For all of these cues to be informative about the location of the middle dot (ie whether it has an exactly central position inbetween the other two dots, or whether it is displaced), we have to assume that the observer knows what the value of this cue is for the standard central position. Because we did not vary the slant of the plane and because observers always received some practice with immediate feedback, it is not unlikely that observers developed some knowledge about this standard value against which they could then evaluate the value of the property for each trial presented in the experiment.

Several problems with the present experimental design make it impossible to assess the use of these cues in more systematic ways. First, the effects of slant and of feedback were not investigated in our experiments. Slant was kept fixed at $45^{\circ}$ and feedback was 
given only in a relatively small number of randomly chosen practice trials. Second, because of the large number of experimental conditions resulting from all combinations of all variables of interest (ie displacement size and direction, segment size, projection type, centre of rotation, display type), there was only a small number of trials in each condition. In principle, it would be interesting to investigate how performance correlates with the variable usefulness of each of the mentioned cues in all of these conditions. However, in the present experiments the number of trials was much too small to assess these covariations systematically. ${ }^{(4)}$ Third, within the range of our stimuli, the correlations between the 3-D displacements and several of the 2-D cues were generally very high. To better assess the role of 3-D versus 2-D sources of information, one would have to extend the range of stimuli by using larger slants, stronger perspective distortions, and larger displacement sizes. In future research, we will design new experiments to overcome these limitations of the present study.

Acknowledgements. The research presented here was supported by grants G.0210.97N and G.0130.98 from the Research Program of the Fund for Scientific Research-Flanders (FWO) to JW. Preliminary reports of this research have been presented at the 19th European Conference on Visual Perception (Wagemans and Tibau 1996), at ARVO (Wagemans and Tibau 1997a), and at the AVA Workshop on Depth Perception (Wagemans and Tibau 1997b). We would like to thank Christian Lamote for programming assistance at the initial stages of this project, Luc Van Gool (Department of Computer Vision) and Paul Dhooghe (Department of Mathematics) for interesting discussions about this research, and Keith Niall and two anonymous reviewers for fruitful comments on previous versions of this paper.

\section{References}

Braunstein M L, Todd J T, 1990 "On the distinction between artifacts and information" Journal of Experimental Psychology: Human Perception and Performance $16211-216$

Carlsson S, Mohr R, Moons T, Morin L, Rothwell C, Van Diest M, Van Gool L, Veillon F, Zisserman A, 1996 "Semi-local projective invariants for the recognition of smooth plane curves" International Journal of Computer Vision 19211 - 236

Cutting J E, 1986 Perception with an Eye for Motion (Cambridge MA: MIT Press/Bradford Books)

Cutting J E, 1991 "Four ways to reject directed perception" Ecological Psychology 3 25-34

Cutting J E, 1996 "Wayfinding from multiple sources of local information in retinal flow" Journal of Experimental Psychology: Human Perception and Performance 22 1299-1313

Cutting J E, Springer K, Braren P A, Johnson S H, 1992 "Wayfinding on foot from information in retinal, not optical, flow" Journal of Experimental Psychology: General 12141 - 72

Eagle R A, Blake A, 1995 "Two-dimensional constraints on three-dimensional structure from motion tasks" Vision Research $352927-2941$

Forsyth D, Mundy J L, Zisserman A, Coelho C, Heller A, Rothwell C, 1991 "Invariant descriptors for 3-D object recognition and pose" IEEE Transactions on Pattern Analysis and Machine Intelligence PAMI-13 971 - 991

Foster D H, Simmons D R, 1994 "Viewpoint invariance of contour-curvature discrimination over extended performance levels" Spatial Vision 8 45-55

Foster D H, Simmons D R, Cook M J, 1993 "The cue for contour-curvature discrimination" Vision Research $33329-341$

Foster D H, Wagemans J, 1993 "Viewpoint-invariant Weber fractions and standard contourcurvature discrimination" Biological Cybernetics 70 29-36

Frisby J P, Buckley D, Duke P A, 1996 "Evidence for good recovery of lengths of real objects seen with natural stereo viewing" Perception 25129 - 154

Hoffman D D, Flinchbaugh B, 1982 "The interpretation of biological motion" Biological Cybernetics $42197-204$

(4) We have tried, but our attempts have failed. When we collapsed data from several conditions and computed average performance levels to regress them against average cue values, results were quite promising (see Wagemans and Tibau 1997a, 1997b). However, when we used the statistically purer method of regressing separate performance levels against separate cue values for all conditions, a very unstable pattern of results emerged that allowed no straightforward interpretation. Clearly, many more data points per condition are needed for this method to be applied with any chance of success. 
Hogervorst M A, Eagle R A, 1998 "Biases in three-dimensional structure-from-motion arise from noise in the early visual system" Proceedings of the Royal Society of London, Series B $2651587-1593$

Johansson G, Jansson G, 1968 "Perceived rotary motion from changes in a straight line" Perception \& Psychophysics $4165-170$

Kim N-G, Growney R, Turvey M T, 1996 "Optical flow not retinal flow is the basis of wayfinding by foot" Journal of Experimental Psychology: Human Perception and Performance 22 $1279-1288$

Koenderink J J, Doorn A J van, 1991 "Affine structure from motion" Journal of the Optical Society of America A 8377 -385

Kubovy M, Holcombe A O, Wagemans J, 1998 "On the lawfulness of grouping by proximity" Cognitive Psychology $3571-98$

Kubovy M, Wagemans J, 1995 "Grouping by proximity and multistability in dot lattices: A quantitative Gestalt theory" Psychological Science $6225-234$

Kukkonen H T, Foster D H, Wood J R, Wagemans J, Van Gool L, 1996 "Qualitative cues in the discrimination of affine-transformed minimal patterns" Perception 25 195-206

Lappin J S, Ahlström U B, 1994 "On the scaling of visual space from motion-in response to Pizlo and Salach-Golyska" Perception \& Psychophysics 55 235-242

Lappin J S, Fuqua M A, 1983 "Accurate visual measurement of three-dimensional moving patterns" Science $221480-482$

Lappin J S, Love S R, 1992 "Planar motion permits perception of metric structure in stereopsis" Perception \& Psychophysics $5186-102$

Niall K K, 1998 "Estimates of shape by eye, or the little invariant that could" Acta Psychologica $100291-320$

Norman J F, Todd J T, 1993 "The perceptual analysis of structure from motion for rotating objects undergoing affine stretching transformations" Perception \& Psychophysics 53 279-291

Norman J F, Todd J T, Perotti V J, Tittle J S, 1996 "The visual perception of three-dimensional length" Journal of Experimental Psychology: Human Perception and Performance 22 173-186

Pizlo Z, Salach-Golyska M, 1994 "Is vision metric? Comment on Lappin and Love (1992)" Perception \& Psychophysics $\mathbf{5 5} 230-234$

Pollick F E, 1997 "The perception of motion and structure in structure-from-motion: Comparisons of affine and Euclidean formulations" Vision Research 37 447-466

Simpson W A, 1986 "The cross-ratio and the perception of motion and structure", in Motion: Representation and Perception Eds N I Badler, J K Tsotsos (New York: North-Holland) pp 248 - 252

Sperling G, Dosher B A, Landy M S, 1990 "How to study the kinetic depth effect experimentally" Journal of Experimental Psychology: Human Perception and Performance 16 445-450

Sperling G, Landy M S, Dosher B A, Perkins M E, 1989 "Kinetic depth effect and identification of shape" Journal of Experimental Psychology: Human Perception and Performance 15 826-840

Tittle J S, Todd J T, Perotti V J, Norman J F, 1995 "Systematic distortion of perceived threedimensional structure from motion and binocular stereopsis" Journal of Experimental Psychology: Human Perception and Performance $21663-678$

Todd J T, Bressan P, 1990 "The perception of 3-dimensional affine structure from minimal apparent motion sequences" Perception \& Psychophysics 48419 -430

Todd J T, Norman J F, 1991 "The visual perception of smoothly curved surfaces from minimal apparent motion sequences" Perception \& Psychophysics $50509-523$

Van Gool L, Moons T, Pauwels E, Wagemans J, 1994 "Invariance from the Euclidean geometer's perspective" Perception $23547-561$

Wagemans J, Tibau S, 1996 "Euclidean cues versus affine or projective invariants in the visual perception of three-dimensional distances" Perception 25 Supplement, 60a (abstract)

Wagemans J, Tibau S, 1997a "Are measured distances in simple moving patterns based on 2-D image properties or 3-D projective invariants?" Investigative Ophthalmology \& Visual Science 38(4) 361 (abstract)

Wagemans J, Tibau S, 1997b "Using multiple sources of information about relative distances between three collinear dots rotating in a slanted plane" Perception 261338 (abstract)

Wagemans J, Van Gool L, Lamote C, Foster D H, 1998 "Minimal information to determine affine shape equivalence" Journal of Experimental Psychology: Human Perception and Performance (manuscript under revision, previous version available as Internal Report No 169), Laboratory of Experimental Psychology, University of Leuven, Leuven, Belgium 